\title{
A comparative study of mulligan's technique vs mulligan combined with dry needling on pain and function in patients with lateral epicondylitis: A clinical trial
}

\author{
Nensi V. Gandhi ${ }^{{ }^{*}}$, Dharmang Vyas ${ }^{2}$, and Lubna Malek ${ }^{3}$ \\ ${ }^{1,2,3}$ Parul Institute of Physiotherapy, Parul University, India \\ *Corresponding E-mail: nensi.modi@paruluniversity.ac.in
}

\begin{abstract}
Background and Purpose: Lateral Epicondylitis is a most common lesion of the elbow affecting the tendinous origin of the wrist extensors especially extensor carpi radialis bravis. The tennis elbow syndrome has been recognized for a century and received its name more than 90 years ago. In clinical practice, different outcome measures were used to evaluate functional recovery in the tennis elbow. This study has used two outcome measures for evaluating the functional recovery in tennis elbow, they are VAS, and Patient rated tennis elbow scale (PRTES). Objectives: To compare the efficacy of Mulligan's technique Versus Mulligan's technique in combination with dry needling therapy, to reduce pain and improve functional disability in lateral epicondylitis. Materials and methods: Subjects with known cases of tennis elbow diagnosed by clinical confirmatory tests by the specialists; were chosen for the study from the Outpatient department of Parul institute of orthopedics department. After dividing the subjects, groups of 20 each, they were pre-tested using PRTES for functional disability and VAS for pain intensity. The first group was given Mulligan's technique along with the conventional exercise and the second group was given Dry needling Therapy and Mulligan's technique along with the conventional exercise 3 times a week for six weeks. The patients were assessed for pain intensity using VAS and functional disability using PRTES was taken by the end of six weeks. The results were computed and analyzed to see which group has better improvement. Results: Thus, there was a significant improvement in functional disability and reduction in VAS in group A and group B. Also there was a significant improvement in functional disability and VAS in group $B$ as compared to group A. Conclusion: In this study, both the groups statistically showed a significant response to their interventions. The mean score in the case of VAS is less and in the case of PRTES is high for Mulligan's technique and dry needling therapy as compared to mulligan's technique alone mulligan's technique and dry needling was found to be more effective than the mulligan's technique alone to reduce the pain and to increase functional disability in treating Lateral Epicondylitis.
\end{abstract}

Keywords: lateral epicondylitis, mulligan`s mobilization with movement, dry needling therapy, visual analogue scale, patient rated tennis elbow scale.

Received: 03 October, 2021

Accepted: 22 October, 2021

Published: 29 October, 2021

\section{Introduction}

Tennis elbow, also known as lateral epicondylitis, is a pathological condition of the wrist extensor muscles at their 
origin on the lateral humeral epicondyl. The tendinous origin of extensor Carpi radials is the area in which overuse or repetitive trauma causes fibrosis and microtears Changes can also be found in musculotendinous structure of extensor Carpi radialis longus, extensor carpi ulnaris, and extensor digitorum communis [1].

Anatomy: The elbow joint is the point of articulation of three bones:

- The humerus of the arm

- The radius

- The ulna of the forearm

Humeroulnar joint is the joint between the trochlea on the medial aspect of the distal end of the humerus and the trochlear notch on the proximal ulna Humeroradial joint is the joint between the capitulum on the lateral aspect of the distal end of the humerus with the head of the radius. A fibrous capsule encloses the joint and is lined internally by a synovial membrane. The elbow joint is functionally a hinge joint, allowing movement in only one plane (uniaxial) [2]. The lateral epicondyle of the humerus is a small, tuberculated eminence, curved a little forward, and giving attachment to the radial collateral ligament of the elbow joint, and to a tendon common to the origin of the supinator and some of the extensor muscles [3]. The medial epicondyle of the humerus is an epicondyle of the humerus bone of the upper arm in humans. It is larger and more prominent than the lateral epicondyle and is directed slightly more posteriorly in the anatomical position. The olecranon is the large, thick, curved bony eminence of the ulna, a long bone in the forearm that projects behind the elbow. It forms the most pointed portion of the elbow and is opposite to the cubital fossa or elbow pit [4].

There are a collection of ligaments that connect the bones forming the elbow joint to each other, contributing to the stability of the joint. The ulnar collateral ligament extends from the medial epicondyle of the humerus to the coronoid process of the ulna. The radial collateral ligament has a low attachment to the lateral epicondyle of the humerus. The annular ligament also reinforces the joint by holding the radius and ulna together at their proximal articulation. The quadrate ligament is also present at this joint and maintains constant tension during pronation and supination movements of the forearm [2].

Prevalence: The prevalence of TE is described to be 1-2\% in a general population between 30 and 64 years of age. The highest incidence is between 40 and 60 years of age and, there are no differences between men and women in occupational populations the prevalence is between $2-23,95 \%$ of tennis elbow occurs in non-tennis players. 10-50 $\%$ of regular tennis players experience tennis elbow symptoms of varying degrees some time in their tennis lives [5].

Etiopathology: The etiology and pathogenesis of TE are not known, but the condition is considered to be an overuse injury of degenerative nature. A theory that repeated microscopic trauma affects the structure in the CEO at the lateral epicondyle, leading to tissue response with oedema and pain, is often proposed in the literature. This tissue response has been interpreted as inflammation, and the condition has been called lateral epicondylitis. However, there is accumulating evidence that there are no signs of a prostaglandin mediated inflammation with involvement of inflammatory cells in the chronic stage (Nirschl, 1992; Regan et al., 1992; Potter et al, 1995; Alfredson et al, 2000) Therefore, the name epicondylitis seems to be a misnomer [5].

Exercise therapy program for tennis elbow: Exercise therapy plays an important role in the conservative management of tennis elbow with treatment frequently advocated for pain control, muscle strengthening and function Intervention consists of elbow muscle strengthening, wrist muscles strengthening, elbow muscles stretching, and wrist muscles stretching Treatment methods such as concentric and eccentric muscle training of 
elbow and wrist and grip strengthening exercise therapy are commonly recommended for patients suffering from tennis elbow.

Mulligan: Mobilization with Movement (Mulligan's Technique) is a modern technique developed by Mulligan for treating lateral epicondylitis. MULLIGAN'S TECHNIQUE is a form of manual therapy that includes a sustained lateral glide to the elbow joint with concurrent physiological movement. This mobilization technique is often used to correct the faulty position of the elbow joint is being widely used in the management of musculoskeletal disorders Miller (2000) described in his case report the use of the MULLIGAN'S TECHNIQUE for lateral epicondylitis resulting in reduced pain, improvement of PFGS, and increased ability to tolerate resisted isometric wrist extension [6].

Dry Needling: When dry needling is incorporated into the treatment plan, results are often seen after 2 or 3 visits. The entire forearm is easily treated with the patient supine on the treatment table, and multiple TrP's can be treated in a matter of minutes. After a thorough history and physical exam, the therapist assesses the forearm for taut bands and trigger points. Muscles are commonly involved in symptoms of lateral.

\section{Vas Scale:}

\section{Purpose}

A Visual Analogue Scale (VAS) is a measurement instrument that tries to measure a characteristic or attitude that is believed to range across a continuum of values and cannot easily be directly measured [8].

\section{Content and response}

It is usually presented as a 100 -mm horizontal line on which the patient's pain intensity is represented by a point between the extremes of "no pain at all" and "worst pain imaginable" Its simplicity, reliability, and validity, as well as its ratio scale properties, make the VAS the optimal tool for describing pain severity or intensity [8].

\section{Administration}

They are generally completed by patient's themselves. The patients marks on the line the point that they feel represents their perception of their current state of pain [8].

\section{Recall period for items}

Recall period for items varies, but most commonly, respondents are asked to report "current" pain intensity or pain intensity "in the last 24 hours [8].

\section{Patient-rated tennis elbow evaluation (PRTEE) scale}

PRTEE is a 15-item questionnaire designed to measure forearm pain and disability in patients with lateral epicondylitis. Reliability and internal consistency of the questionnaire were excellent (PRTEE pain subscale, 0.94; PRTEE specific activities subscale, 0.93; PRTEE usual activities, 0.85) Correlations were good between the PRTEE subscales and total scale and the VAS and DASH Standardized response means (SRM) were higher in the PRTEE $(\mathrm{SRM}=2.1)$ than in the other outcome measures (SRM, 1.5-1.7). The PRTEE was a reliable, reproducible, and sensitive instrument for the assessment of chronic lateral elbow tendinopathy in a tennis-playing cohort [9].

\section{Scoring}

Pain Subscale- Add up 5 items: Best score $=0$; Worst score $=50$ 
Specific Activities- Add up 6 items: Best Score= 0; Worst Score= 60 .

Usual Activities- Add up 4 items: Best Score=0; Worst Score $=40$.

Function Subscale- (Specific Activities + Usual Activities $) / 2$ : Best score $=0 ;$ Worst score $=50$.

Total Score $=$ Pain Subscale + Function Subscale: Best Score $=0$; Worst Score $=100$ (pain and disability contribute equally to score) [9].

\section{Materials and method:}

Source of data: Patients suffering from lateral epicondylitis coming to physiotherapy clinics/centers in and around Vadodara.

Method of collection of data: Approximately 40 subjects with the age group of 20-60 years, fulfilling the inclusion criteria and exclusion criteria were taken into the study and assigned into the two groups; group A and group B. Duration of the study was approximately 3 weeks.

\section{Inclusion criteria:}

- Unilateral symptomatic lateralepicondylitis.

- Both Male and Female.

- Subjects with age group 20-60years.

- Tenderness over the forearm extensor origin.

- Pain on the lateral epicondyle during resisted dorsiflexion of the wrist with the elbow in full extension.

\section{Exclusion criteria:}

- Subjects with bilateral lateral epicondylitis

- History of Rheumatoid diseases or Neurologic impairment including Stroke or Head injury; Severe Neck Shoulder problem likely to cause or maintain elbow complaints

- History of fracture of humerus or radius or ulna; Intra-articularpathology/hematoma

- Arthritis or allied conditions; Elbow bursitis; Medial epicondylitis; Radial tunnel syndrome; Cervicalradiculopathy

- Skin problems/neighboring bacterial infection; Ossification and calcification of the soft tissues

- Previous surgery to elbow joint; treated previously by physiotherapy or any other kind of manual therapy in the last 3 weeks before inclusion

- Non-co-operative patients

Subjects meeting inclusion and exclusion criteria were recruited for the study. Informed consent was obtained from them comparative study design is used. Informed consent was obtained from the comparative study design is used. The subjects were randomly assigned into two groups, group A and group B with 20 subjects in each group. Pretreatment assessment on VAS for pain and PRTES for functional disability was noted for both groups.

Group A subjects underwent Mulligan`s technique.

Group B subjects underwent the Mulligan`s technique and Dry needling Therapy.

Frequency of the treatment regimen for both the groups was three times a week for a period of 3 weeks. On the 
same assessment parameters, Post-treatment assessments of Pain and functional disability were taken for both the groups by the end of the third week. The results were computed and analyzed to see which group has better improvement.

\section{Outcome Measures}

The outcome is measured in terms of pain and functional disability, using VAS and PRTES respectively at the following intervals:

- At baseline

- At end of the third week after starting treatment

\section{Procedure for measuring visual analogue scale}

Patient was provided with a visual analogue scale (VAS) The VAS used in the study consisted of a continuous horizontal line $10 \mathrm{~cm}$ in length with anchor point of 'no pain' (0) and 'worst pain' (10) on the left and right ends of line respectively [69]. Patient was explained before the treatment about VAS with respect to 0-10 on the scale. The patients were asked to mark pain intensity before the treatment and by the end of the third week.

\section{Procedure for measuring functional disability by using PRTES}

PRTES is commonly used for Functional Disability measurement in clinical and research settings.

During the examination, the patient was seated comfortably and PRTES scale was explained to patients. It is a 15 items questionnaire designed to measure forearm pain and disability in patients with tennis elbow consist so subscales, where pain subscale- $0=$ no pain to $10=$ worst pain, functional subscale- $0=$ no difficulty to $10=$ unable to do. Total score is 100 according to level of activity of the patient's marking was done.

\section{Intervention}

Subjects were randomly assigned to two groups Informed consent (fig 3.3) was obtained from them.

\section{Group A}

Subjects received Mulligan`s Mobilization with Movement. Subjects were instructed to lie supine having their elbow extended and forearm pronated on a treatment table Belt was put around patient forearm With the patient established what active motion reproduced the patient's elbow pain; this was considered to be the 'comparable sign The comparable sign was one of the following: making a fist, gripping a rolled elastic bandage of $5 \mathrm{~cm}$ diameter, wrist extension un- resisted, and wrist extension resisted third finger extension un-resisted, or third finger extension resisted If any of these motions reported as painful was designated the comparable sign, and no further motions were assessed. Then mulligan's technique was performed, consisting of a laterally-directed manual pressure to the proximal medial forearm while the subject performed the comparable sign motion (Mulligan 1995) Based on the suggestion of Mulligan (1995), up to four attempts were allowed to find the direction of the manual pressure that eliminated the comparable sign on the affected side. The four directional options were standardized and recorded on the data format this time if pain with the comparable sign was eliminated (positive response to mulligan's technique) if pain with the comparable sign was not eliminated (negative response to mulligan's technique) based on 
the suggestion of mulligan (1995), the patient performed previously painful motion up to ten times while mulligan's technique was being applied and sustained for approximately thirty seconds. It was done for three sets with thirty seconds rest in between each set.

\section{Group B}

Subjects received Dry needling Therapy followed by Mulligan`s Mobilization with Movement Dry Needling was given needles size $25 \mathrm{~mm}$ or $13 \mathrm{~mm}$ size.

Dry needling is the invasive procedure performed to release the pain and improve the movement. After the through history and physical examination the therapist assess the forearm for taut bands and trigger points. Therapist wears gloves and sterilizes his hands and the part to be treated. Needle is being held with the middle finger and the thumb perpendicular to the muscle to be treated and therapist uses index finger to tap insert the needle into the skin and then after removing guide tube full-length needle is inserted inside the muscle. It can be kept for 10 minutes up to half-hour then after this time needle is gently removed pressing the surrounding skin and rubbing alcohol is dabbed over it for some time.

\section{Pre-treatment measurement}

For Pre-treatment assessment, Pain and Functional Disability were noted for both the groups. VAS and PRTES were used to measure pain and Functional Disability respectively.

\section{Post-treatment measurement}

On the same assessment parameters, Post-treatment assessments of Pain and functional disability were taken, at the end of 3 weeks, for both groups. The readings of a range of motion for each subject were recorded in an evaluation chart (Refer Annexure). The readings of all the subjects are tabulated in the master chart and taken up for statistical analysis.

\section{Exercise therapy for tennis elbow}

Conventional exercise protocol for lateral epicondylitis is common in both groups. This includes strengthening \& stretching of elbow \& wrist musculature for the duration of 3 weeks in both groups. Strengthening of the elbow and wrist muscles was done with the progression of theraband on weekly basis.

\section{Wrist extensors strengthening with theraband}

Patient lies in sitting position with back supported on chair and pillow is kept on the lap of the patient for forearm support Patient holds the theraband from the unaffected hand and affected hand of the patient is semi flexed with forearm prone and supported on the pillow and then keeping the theraband straight patient moves the wrist of the affected forearm in the upward direction (extension) 15 repetitions are done in one sitting for the period of 3 weeks for 3times/weekly.

\section{Wrist flexors strengthening with theraband}

Patient lies in a sitting position with the back supported on a chair and the pillow is kept on the lap of the patient for 
forearm support. Patient holds the theraband from the unaffected hand and the affected hand of the patient is semi flexed with forearm supine and supported on the pillow and then keeping the theraband straight patient moves the wrist of the affected forearm in the downward direction (flexion). 15 repetitions are done in one sitting for the period of 3 weeks for 3times/weekly. Elbow extensors strengthening with theraband. Patient is in a sitting position on stool and the therapist is standing behind the patient therabnd one end is given in patient's hand and the other end is in the therapist's hand patient's affected forearm is semi flexed and she is asked to extend the elbow against the resistance provided by the theraband given for 15 rep for 3 weeks 3times/weekly.

\section{Statistical analysis}

\section{Age-wise distribution in both the groups:}

Distribution of subjects of groups A and B depicts that the age group of the $2^{\text {nd }}$ decade is most affected with $63 \%$ of the subjects in the group.

\section{Gender wise distribution in both the groups:}

There were $45 \%$ males in groups A and 55\% females in group B there were $60 \%$ males and $40 \%$ females. There was a difference in the number of the males and females distributed in both the groups.

There were $45 \%$ males in group A and 55\% females. In group B there were $60 \%$ males and $40 \%$ females. There was a difference in the number of males and females distributed in both the groups.

There were $45 \%$ males in group A and 55\% females. In group B there were $60 \%$ males and $40 \%$ females. There was a difference in the number of males and females distributed in both the groups. The difference between the pre and post-test values of VAS in group A. The mean value of VAS before treatment was 6.50 with SD 0.946 and after treatment was 3.00 with SD 1.076, Z Value is 3.998 and the p-value is 0 . This shows that there is a highly significant difference between the pre and post-test value of VAS in group A. The difference between the pre and post-test values of VAS in group A. The mean value of VAS before treatment was 650 and after treatment, it was 3.00

\section{Result:}

Total fourty subjects were included in the study after satisfying the inclusion criteria. Group A consists of twenty subjects in which $45 \%$ were males and $60 \%$ were females and group B consists of twenty subjects in which $55 \%$ were males and $40 \%$ were females. Hence males and females were randomly distributed in both groups. Based on age the 4 th decade was mostly effected group in this study by $63 \%$.

In group A, pre-treatment mean VAS was 6.5, and post-treatment it was 3.0. The pre-treatment mean VAS for group B was 6.4 and post-treatment was 2.9. The mean difference of pre vs post-test values of VAS among the groups was 3.0 A and 3.5 for group B. This shows that group B showed a better response to the combined treatment with a pvalue was 0.0001 which shows that there is a highly significant difference of VAS value betweesn group A and group B

There was a highly significant difference between the pre and post-test values of PRTES group A as the p-value was 0.0001 and wilcoxen test value was (z value) 3.933 . 


\section{Discussion}

Lateral epicondylitis is arguably the most common painful, debilitating and upper extremity musculoskeletal disorder [1].

Lateral Epicondylitis has been demonstrated to occur in up to $50 \%$ of tennis players the typical patient is a man or woman aged 30-55 years who either is a recreational athlete or one who engages in rigorous daily activities.

The anatomic basis of the injury to the extensor carpi radialis brevis origin appears to be multifaceted, involving hypovascular zones, eccentric tendon stresses, and a microscopic degenerative response.

Upon examination, the patient has a point of maximal tenderness just distal $(5-10 \mathrm{~mm})$ to the lateral epicondyle in the area of the ECRB muscle. Wrist extension or supination (but not flexion or pronation) against resistance with the elbow extended should provoke the patient's symptoms.

Although many treatments have been advocated, which modality works best, for both conservative and operative treatment choices is still under debate.

\section{Conclusion}

In this study, taking into consideration the two parameters pain and functional disability using VAS and PRTES score respectively was determined in both the groups. Group B comprising of combined treatment of Dry Needling and Mulligan's Technique showed a better result in improvement of functional disability and decline in VAS.

\section{DECLARATIONS}

\section{Funding}

This research did not receive any specific grant from funding agencies in the public, commercial, or not-for-profit sectors.

\section{Conflicts of Interest}

The authors declared no potential conflicts of interest with respect to the research, authorship, and/or publication of this article.

\section{REFERENCES}

[1] Alexander J. Chien et Al.1 Sonography and MR Imaging of Posterior Interosseous Nerve Syndrome with Surgical Correlation. The American Journal of Roentgenology, number 1, volume 181, July 2003, p219-221. Level of Evidence:2C

[2] Van Rijn RM, Huisstede BM, Koes BW, BurdorfA. Associations between work-related factors and specific disorders at the elbow: a systematic literature review. Rheumatology (Oxford). May 2009;48(5):528-36.A1

[3] Dommerholt, J. and Gerwin, R., Trigger Point Needling Course Manual, The Janet Travell, MD Seminar Series,2006

[4] TuomoPienimäki, TuulaTarvainen, PerttiSiira, Antti Malmivaara, HeikkiVanharanta The Clinical journal of pain 18 (3), 164-170,2002

[5] Bisset L, PaungmaliA, Vicenzino B, Beller E. A systematic review and meta-analysis of clinical trials on physical interventions for lateral epicondylalgia. Br J Sports Med 2005 Jul; 39(7):411-22. 
[6] Brukner P, Khan K. Clinical Sports Medicine, Sydney: McGraw-Hill;1993.

[7] Vicenzino B, Wright A. Effect of a novel manipulative physiotherapy technique on tennis elbow: a single study. Manual therapy $1995 ; 1: 30-35$.

[8] HutsonAM.Work-RelatedUpperLimbDisorders:RecognitionandManagement.1997;54

[9] Abbott JH, PatlaCE, Jensen RH. The initial effects of an elbow mobilization with movement technique on grip strength in subjects with lateral epicondylalgia. Manual Therapy 2001; 6(3): 163-169.

[10] Vicenzino B, PaungmaliA, Buratowski S, Wright A. Specific manipulative therapy treatment for chronic lateral epicondylalgia produces uniquely characteristic hypoalgesia. Manual Therapy 2001; 6(4):205-212.

[11] Vicenzino B. Lateral epicondylalgia: a musculoskeletal physiotherapy perspective. Manual Therapy 2003; 8(2):6679.

[12] Manchanda G, Grover D. Effectiveness of movement with mobilization compared with manipulation of wrist in case of lateral epicondylitis. Indian Journal of Physiotherapy and Occupational 2008; 2(1).

[13] Norris C. Sports injuries - diagnosis and management, Butterworth Heinemann 3rded 2005; $412-417$. 